Apidologie, 1976, 7 (4) 277-300.

\title{
RÉCOLTE DES POLLENS ET APPROVISIONNEMENT DU NID CHEZ OSMIA COERULESCENS L. (HYMENOPTERA, MEGACHILIDAE)
}

\author{
Pollenernte und Versorgung des Nestes mit Pollen bei \\ Osmia coerulescens $L$. \\ (Hymenoptera, Megachilidae)
}

J.-N. TASEI

avec la collaboration technique de M. PICART et 5 CARRE

Laboratoire de Zoologie I.N.R.A.

86600 Lusignan

SUMMARY

\author{
POLLEN COLLECTION AND NEST PROVISIONING BY \\ Osmia Coerulescens \\ (Hymenoptera Megachilidae)
}

\begin{abstract}
Analysis of the contents of 373 Osmia coerulescens cells taken from trap nests in the Lusignan area over four years yielded information on the plants visited for pollen collection by this solitary bee for different sites and periods of the year.

Females collect pollen from about 30 plant species belonging to 10 families. On an average $66 \%$ by volume of provisions are from Papilionaceae : Trifolium repens, $T$. pratense, Medicago sativa, Lotus corniculatus; $31 \%$ from Labiatae : Calamintha clinopodium, Ballota foetida; $3 \%$ from other plants : Linaria vulgaris, Papaver rheas, Verbena officinalis, Hypericum perforatum, mostly collected by females of the second annual generation.

Each trapping site is usually characterized by two principal pollens constantly collected. In most cases, 2-5 floral species are represented in a provision. Unifloral provisions involve the most abundant Papilionaceae. The pollen spectrum of a solitary bee species can be established from the analysis of 30 pollen provisions, provided that they are sampled over the whole flight period and in several sites. Osmia coerulescens females load their scopa with pollen in about 9 minutes and unload in the nest in one minute.
\end{abstract}




\section{RÉSUMÉ}

L'examen du contenu de 373 cellules d'Osmia coerulescens récoltées par piègeage dans la région de Lusignan au cours de 4 ans a permis de savoir quelles sont les plantes visitées pour le pollen par cette abeille solitaire en différents lieux et à différentes périodes de l'année.

Les femelles récoltent le pollen sur une trentaine d'espèces de plantes appartenant à une dizaine de familles. En moyenne $66 \%$ du volume des provisions sont composées par des Papilionacées : Trifolium repens, T. pratense, Medicago sativa, Lotus corniculatus; $31 \%$ par des Labiées: Calamintha clinopodium, Ballota foetida; $3 \%$ par d'autres plantes : Linaria vulgaris, Papaver rheas, Verbena officinalis, Hypericum perforatum, récoltées surtout par les individus de la $2^{\mathrm{c}}$ génération annuelle.

Chaque site de piègeage est généralement caractérisé par deux pollens principaux constamment récoltés. Le plus souvent 2 à 5 espèces florales sont représentées dans chaque provision. Les provisions uniflorales sont composées par les pollens des Papilionacées les plus abondantes. Le spectre pollinique d'une espèce peut être établi après l'analyse d'un échantillon de trente provisions pourvu qu'elles soient prélevées sur toute la période d'activité et en plasieurs endroits. Les femelles d'Osmia coerulescens remplissent leur brosse de pollen en $9 \mathrm{mn}$. et se libèrent de leur charge en passant en moyenne $]$ mn dans le nid.

\section{INTRODUCTION}

Les osmies ont été longtemps considérées comme des curiosités par les spécialistes du comportement animal, mais depuis quelques années on pense que certaines espèces peuvent être domestiquées et utilisées en champ ou en verger afin d'assurer une bonne pollinisation chez des espèces végétales peu ou irrégulièrement fréquentées par l'Abeille domestique.

Pour l'instant, quatre espèces retiennent l'attention des chercheurs : Osmia cornifrons Rad., O. lignaria Say., O. rufa L. et $O$. coerulescens L. La première de ces osmies a déjà fait ses preuves en verger de pommes au Japon (Yamada et al., 1971). Aux U.S.A. Torcho (communication personnelle) achève la mise au point du premier élevage c industriel » d'O. lignaria en verger. En Angleterre, Free et Williams (1970) considèrent qu'O. rufa L. peut être domestiquée pour polliniser les plantations commerciales de Rubus et Houm (1973) au Danemark suggère d'utiliser cette même espèce en verger. Enfin $O$. coerulescens qui est un pollinisateur potentiel de la luzerne a déjà fait l'objet d'un travail préliminaire (TASEI, 1972).

L'utilisation en agriculture des espèces pollinisatrices sauvages a généralement débuté après une série d'études approfondies sur le comportement de nidification, les facteurs de la dynamique des populations etc. Les résultats que j'expose plus loin ont été obtenus après quatre ans d'étude sur le comportement de butinage, la récolte du pollen et l'approvisionnement des cellules chez Osmia coerulescens.

\section{MATÉRIEL ET MÉTHODES}

Deux types d'études sont réalisées ici : le premier est un inventaire des pollens récoltés par $O$. coerulescens nidifiant dans des pièges à bambous placés dans la nature; le second est l'observation de l'activité de butinage et d'approvisionnement de quelques femelles élevées en enceinte fermée. 
a) Cinquante à cent vingt nichoirs suivant l'année sont accrochés contre des parois de bâtiments et des poteaux en bois susceptibles d'être des sites naturels de nidification (cf. : TASEI, 1972). Le dispositif est maintenu pendant quatre années de 1970 à 1973 , dans un rayon de $10 \mathrm{~km}$ autour de Lusignan (Centre-Ouest de la France). Au cours de cette période un total de 373 prélèvements de pollen et d'analyses microscopiques sont effectués. L'échantillonnage est hebdomadaire pendant toute la saison d'activité de cette osmie, d'avril à septembre.

b) Par ailleurs, l'élevage de 8 femelles a lieu dans des compartiments d'une serre vitrée. Pendant 21 heures au total, l'activité de butinage et d'approvisionnement de ces insectes est observée et chronométrée. Plusieurs espèces végétales en fleur sont fournies simultanément aux femelles.

Lorsqu'un voyage est le premier d'une série d'approvisionnements on prélèvera le chargement de pollen dès qu'il est déposé au fond de la cellule puis on l'analyse. Le total de tels échantillons correspond à 7 heures de butinage.

La technique de montage des échantillons de pollen (cf. : Maurizio et Louveaux, 1965), la méthode de détermination des espèces et la correction volumétrique des proportions numériques des différents pollens sont les mêmes que celles qui sont appliquées dans deux travaux précédents (TASEI, 1972 et 1973).

TARL. 1. - Fréquence de la présence des espèces de pollens récoltés par Osmia coerulescens.

TAB. 1. - Häufigkeit der von Osmia coerulescens eingetragenen Pollenarten.

\begin{tabular}{|c|c|c|c|}
\hline \multicolumn{2}{|c|}{$\begin{array}{c}\text { Espèces de Pollen } \\
\text { Pollenarten }\end{array}$} & $\begin{array}{l}\text { Nombre de cellules } \\
\text { renfermant l'espèce } \\
\text { de pollen } \\
\text { Anzahl d. Zellen, } \\
\text { die die Pollenart } \\
\text { enthalten }\end{array}$ & $\begin{array}{c}\text { Pourcentage du total } \\
\text { des cellules (373) } \\
\text { Prozentsatz der } \\
\text { Gesamtzellenzahi } \\
\end{array}$ \\
\hline 总 & 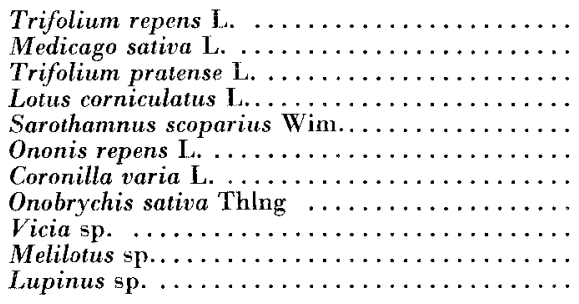 & $\begin{array}{r}166 \\
153 \\
131 \\
127 \\
36 \\
14 \\
11 \\
8 \\
8 \\
3 \\
1\end{array}$ & $\begin{array}{r}44,6 \\
41,1 \\
35,2 \\
34,1 \\
9,7 \\
3,8 \\
2,9 \\
2,1 \\
2,1 \\
0,8 \\
0,3\end{array}$ \\
\hline 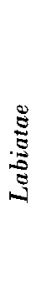 & 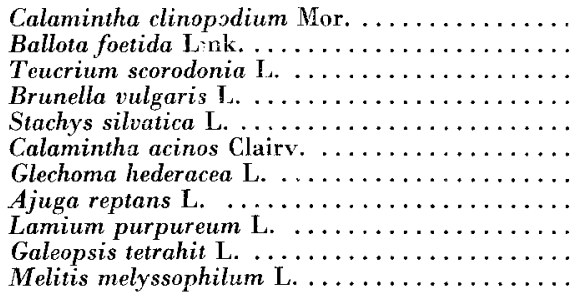 & $\begin{array}{r}117 \\
113 \\
66 \\
56 \\
42 \\
10 \\
6 \\
5 \\
3 \\
1 \\
1\end{array}$ & $\begin{array}{r}31,4 \\
30,4 \\
17,7 \\
15,0 \\
11,3 \\
2,7 \\
1,6 \\
1,3 \\
0,8 \\
0,3 \\
0,3\end{array}$ \\
\hline 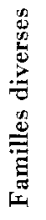 & 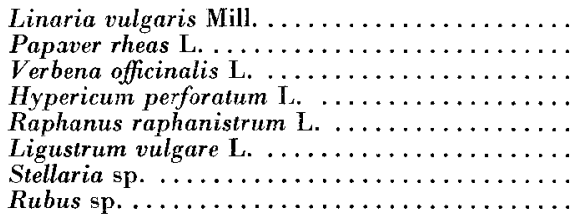 & $\begin{array}{r}26 \\
16 \\
13 \\
12 \\
2 \\
1 \\
1 \\
1\end{array}$ & $\begin{array}{l}7,0 \\
1,3 \\
3,5 \\
3,2 \\
0,5 \\
0,3 \\
0,3 \\
0,3\end{array}$ \\
\hline
\end{tabular}




\section{RÉSULTATS}

I. - La récolte du pollen dans les conditions naturelles

a) L'inventaire des pollens dans l'ensemble des cellules échantillonnées pendant 4 ans.

Les 373 analyses de provisions donnent le spectre de récolte moyen (tabl. 1, fig. 1) : trente espèces végétales réparties en 10 familles sont visitées pour le pollen. Deux familles de plantes sont très bien représentées : les Labiées et surtout les Papilionacées. Six espèces sur 30 se rencontrent dans plus de 30 pour cent des provisions : Trifolium repens L., Medicago sativa L., Trifolium pratense L., Lotus corniculatus L., Calamintha clinopodium Mor., Ballota foetida Lmk. La fréquence de rencontre des pollens appartenant à d'autres familles est assez faible : quatre espèces sont relativement bien représentées Linaria vulgaris Mill. (7 pour cent), Papaver rheds L. (4 pour cent), Verbena officinalis L. ( 3 pour cent), Hypericum perforatum L. (3 pour cent).

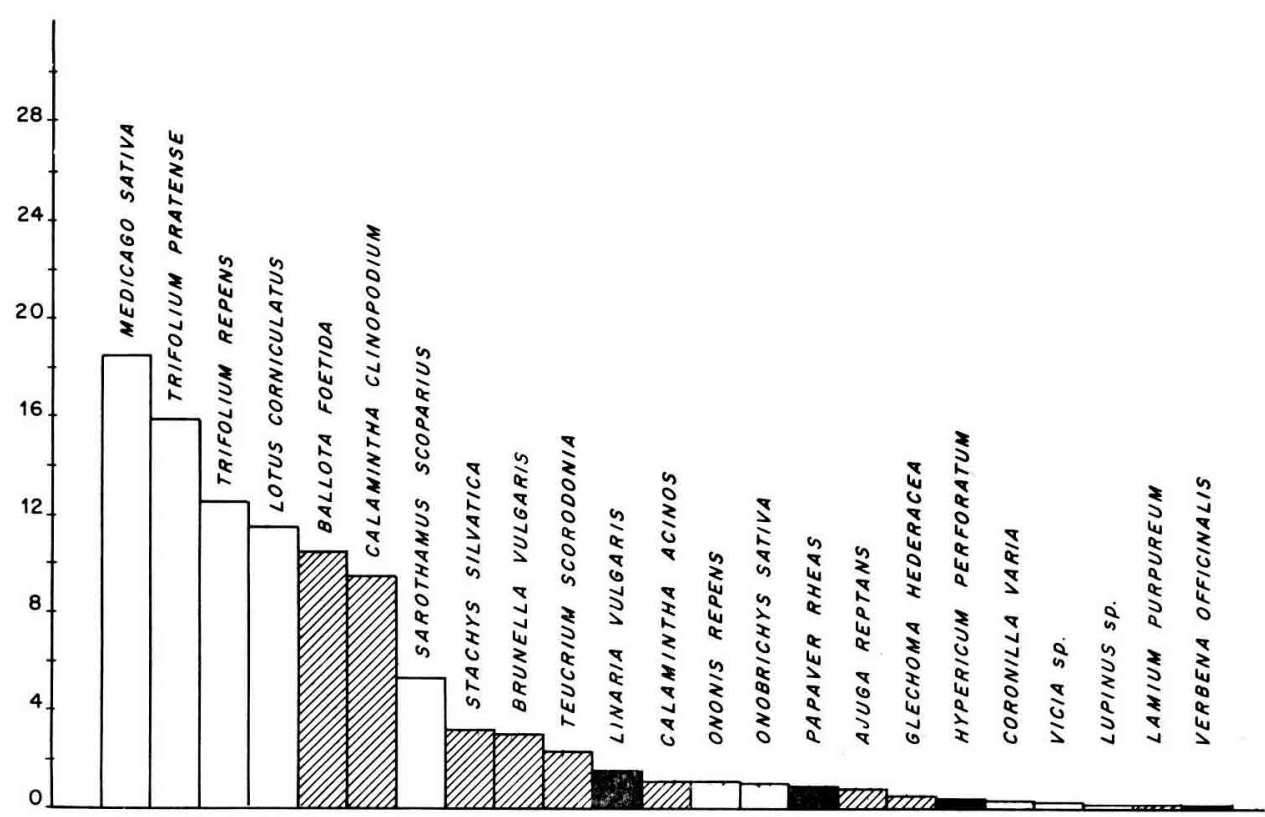

FIG. 1. - Spectre de récolte des pollens par Osmia coerulescens à Lusignan au cours de 4 années (373 cellules).

Verticalement : pourcentage du volume total de la récolte de pollen.

Авв. 1. - Spektrum der Pollenernte von Osmia coerulescens in Lusignan im Verlauf von vier Jahren (373 Zellen)

Ordinate $=\%$ des Gesamtvolumens der Pollenernte 
Après comptage des grains de pollen et correction volumétrique on obtient le spectre de récolte de façon précise (fig. 1). Cette figure fait ressortir l'importance des quatre Papilionacées et des deux Labiées citées plus haut. D'une façon générale on peut dire qu'il y a correspondance entre la figure 1 et le tableau 1, e'est-à-dire que lorsqu'un pollen apparaît dans un grand nombre d'échantillons il représente un volume plus considérable qu'un pollen moins fréquent.

b) Les variations de la récolte de pollen selon l'année

Quelle que soit l'année d'observation les Papilionacées représentent toujours plus de 50 pour cent du volume total des pollens récoltés (53 à 89 pour cent), les Labiées 31 pour cent ( 11 à 41 pour cent) et les autres plantes 3 pour cent $(0,2$ à 5 pour cent) (tabl. 2$)$.

TABL. 2. - Pourceatages des quantités (estimation volumétrique) de pollen de Labiatae et Papilionaceae récoltées par Osmia coerulescens à Lusignan.

TAB. 2. - \% der Pollenmengen (volumetrische Schätzung) von Labiaten und Papilionaceen, die von 0 . coerulescens in Lusignan eingetragen wurden.

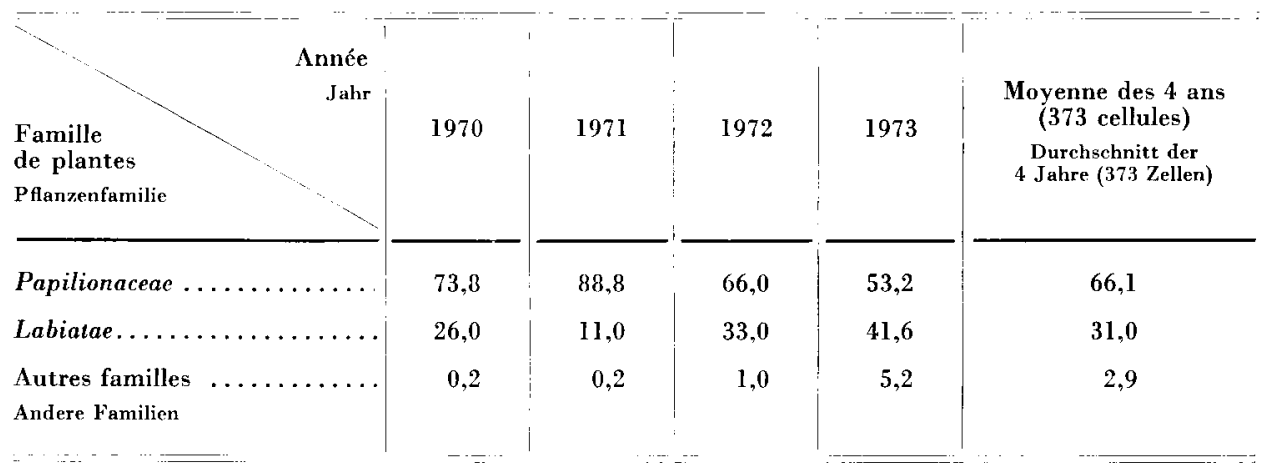

c) Les variations du spectre de récolte en fonction du lieu

Malgré un échantillonnage irrégulier car dépendant de l'abondance locale de l'espèce (tabl. 3) on donne sur la figure 2 les spectres de récolte de pollen partiels par lieu de piegeage et par année. On remarque que les osmies ont tendance à récolter en un même lieu, les mêmes pollens principaux, par exemple :

- Medicago sativa et Trifolium repens et secondairement Calamintha clinopodium dans la localité : Verrines.

- Medicago sativa dans la localité : Chêne.

- Trifolium pratense et Calamintha clinopodium dans la localité : Pétinière.

— Lotus corniculatus et Sarothamnus scoparius dans la localité : Mongadon. 
TABL. 3. - Nombre de cellules d'Osmia coerulescens ayant fait l'objet d'une analyse pollinique suivant l'année et les 4 principaux lieux de piègeage aux environs de Lusignan.

TAB. 3. - Anzahl der Zellen von 0 . coerulescens, die pollenanalytisch nach Jahren an den vier hauptsächlichen Nistplätzen in der Umgebung von Lusignan untersucht wurden.

\begin{tabular}{|c|c|c|c|c|c|}
\hline Oreu & 1970 & 1971 & 1972 & 1973 & Tota \\
\hline Verrines...... & - & 42 & 6 & 17 & 65 \\
\hline Chêne. & 3 & 23 & 7 & 41 & 74 \\
\hline Pétinière ... & 10 & 18 & 25 & 45 & 98 \\
\hline Mongadon ... & 11 & 22 & 4 & 11 & 48 \\
\hline & 24 & 105 & 42 & 114 & 285 \\
\hline
\end{tabular}

Ces spectres de la récolte de pollen sont cependant très variables, puisqu'une année donnée certaines espèces apparaissent tandis que d'autres disparaissent.

d) Les variations de la récolte en fonction du moment de l'année

Le tableau 4 rend compte de la variabilité de l'échantillonnage qui est due à l'échelonnement des sorties des abeilles en début de période et aux différences de climat d'une année à l'autre. Les figures 3,4 et 5 donnent le détail des analyses hebdomadaires de pollen pour 3 années. Le tableau 5 condense ces résultats et indique la diversité du spectre moyen selon le mois de l'année. Juin, juillet et août sont les mois pendant lesquels on peut trouver le plus d'espèces, par exemple 19 espèces sur 30 sont rencontrées en juillet. Ces pollens appartiennent à 8 familles botaniques. Les 2 seules familles à être constamment représentées de mai à septembre sont les Papilionacées et les Labiées. Enfin, 3 familles offrent leur pollen de façon tardive : les Hypericacées (Hypericum perforatum), les Rosacées (Rubus sp.) et les Verbenacées (Verbena officinalis) et seules les femelles de deuxième génération peuvent en profiter.

e) Les associations de pollens dans les cellules

Sur 373 provisions analysées, 50 sont des provisions uniflorales (tabl. 6). Près de 200 sont composées d'apports provenant de 2 et 3 espèces florales. Plus de 100 sont des mélanges de 4 à 5 espèces. Dans 16 provisions seulement on trouve 6 ou 7 espèces en mélange. 
RÉCOLTE DES POLLENS CHEZ osmia COERULEscens

PAPILIONACEAE

LABIATAE

DIVERS
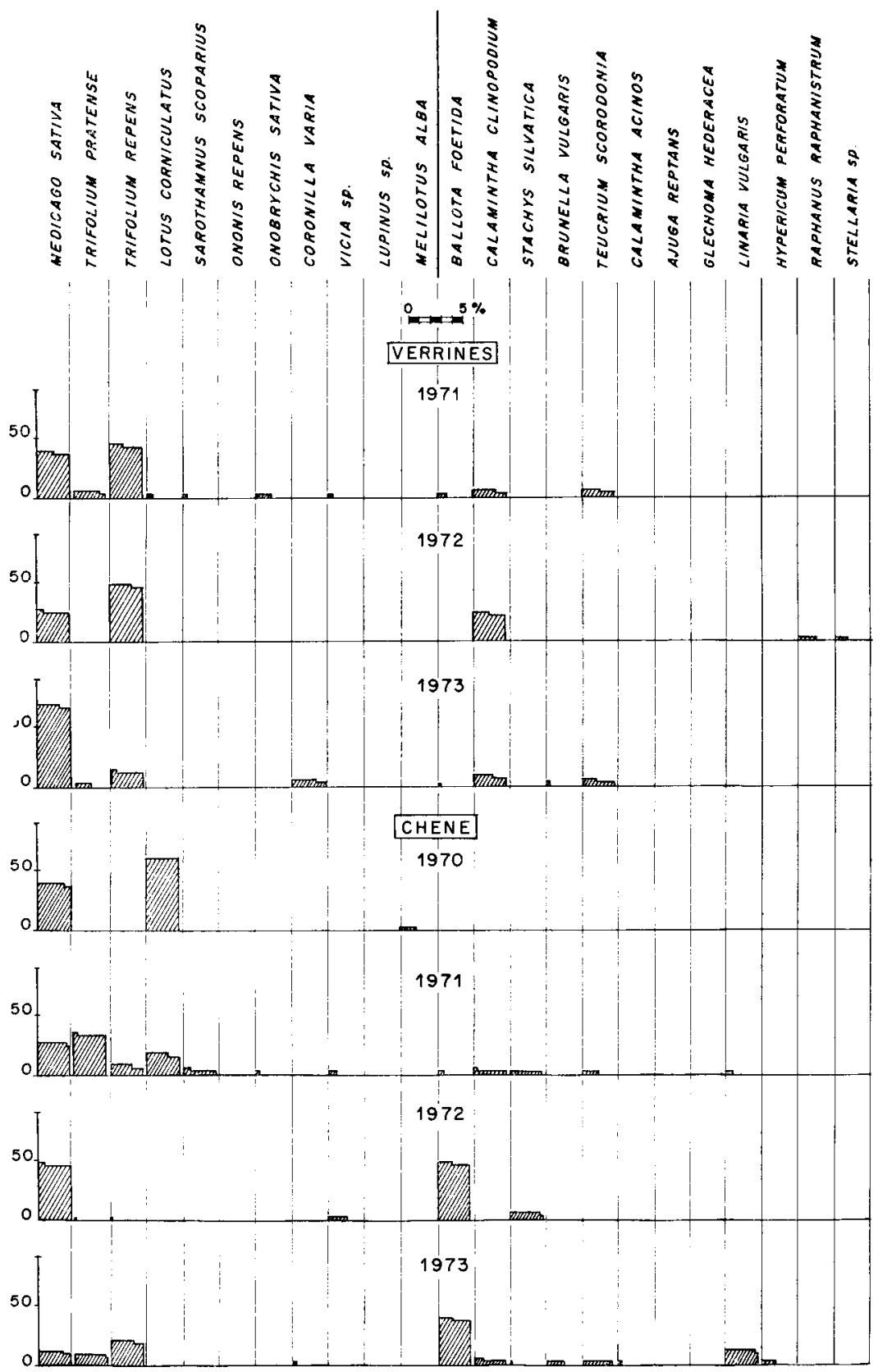

Fig. 2 (A). - Spectre de récolte des pollens par Osmia coerulescens dans 4 lieux aux environs de Lusignan.

Verticalement : Pourcentage du volume des pollens récoltés dans chaque lieu.

Авв. 2 (A). - - Spektrum der Pollenernte von 0 . coerulescens an 4 Plätzen in der Umgebung von Lusignan

Ordinate $=\%$ der an jedem Ort gesammelten Pollenmenge 

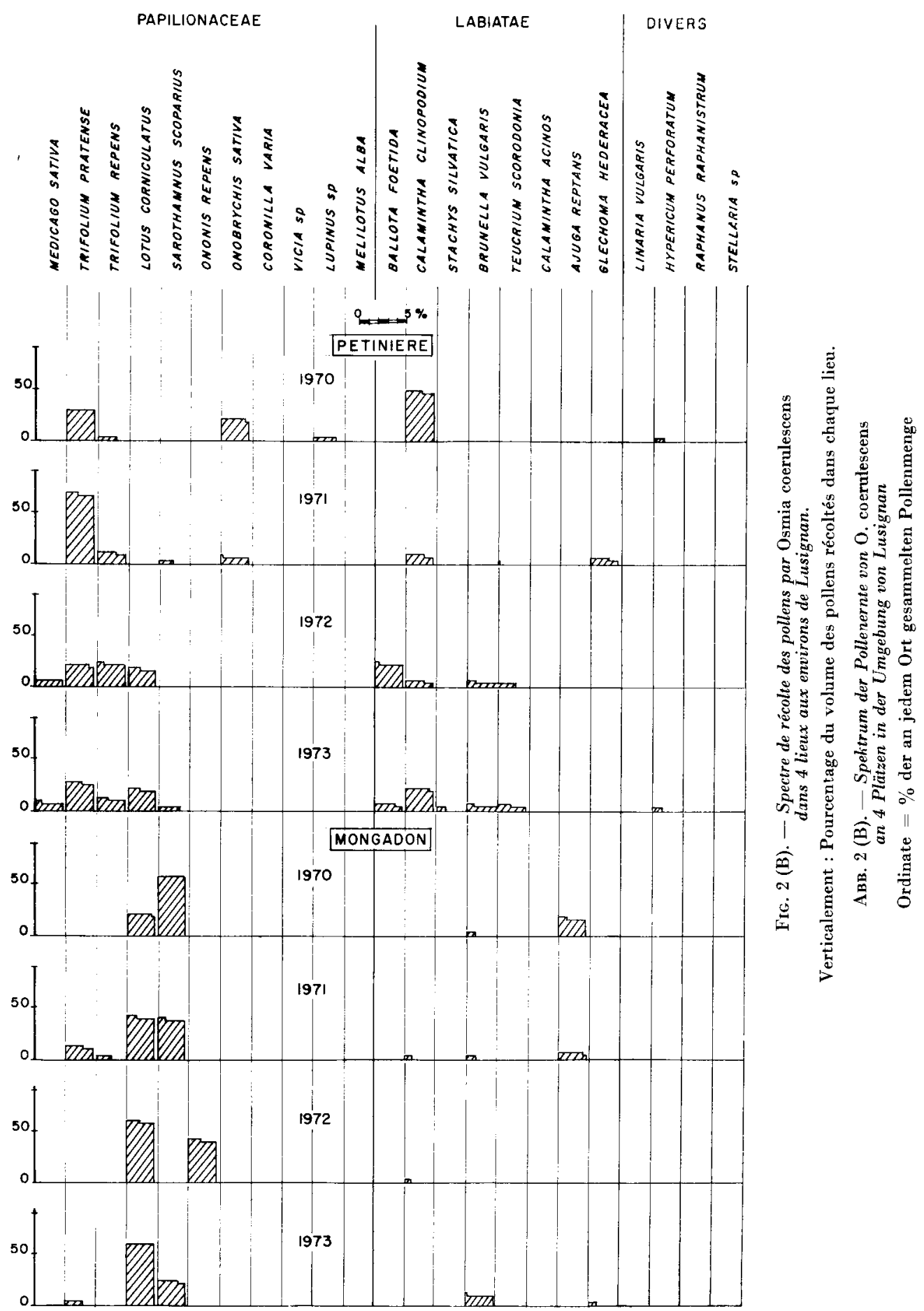
TABL. 4. - Nombre de cellules d'Osmia coerulescens ayant fait l'objet d'une analyse pollinique suivant l'année et le mois dans tous les lieux de piègeage de Lusignan.

ТАВ. 4. - Anzahl der Zellen von 0 . coerulescens, die pollenanalytisch nach Jahren und Monaten an allen Plätzen von Lusignan untersucht wurden.

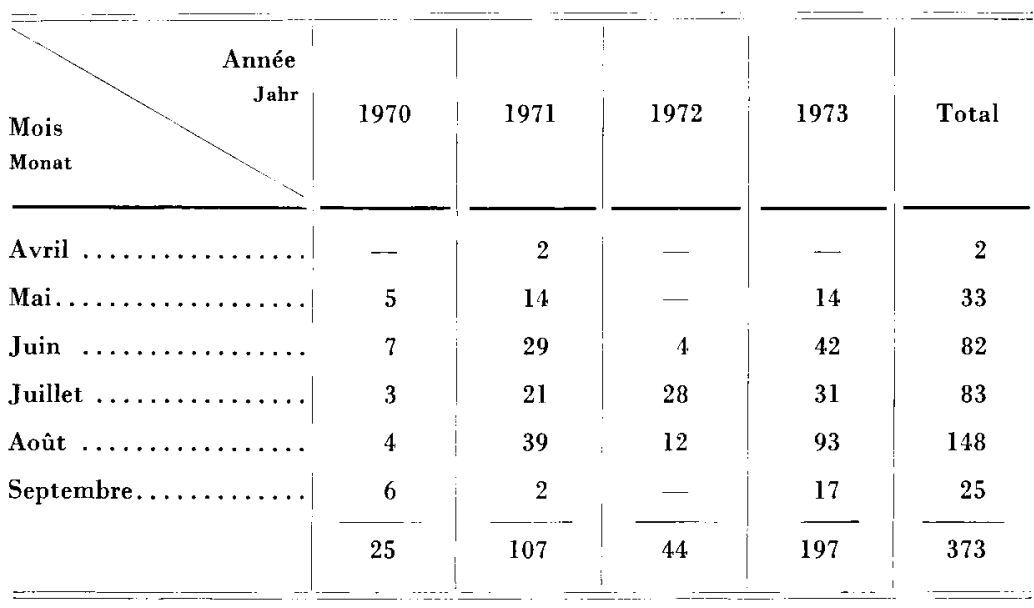

TaBl. 5. - Diversité des pollens récoltés par Csmia coerulescens en fonction du mcis, au cours de 4 ans à Lusignan.

TAB. 5. - Verschiedenheit der von 0 . coerulescens im Verlauf von vier Jahren in Lusignan gesammelten I'ollenarten in Abhängigkeit vom Sammelmonat.

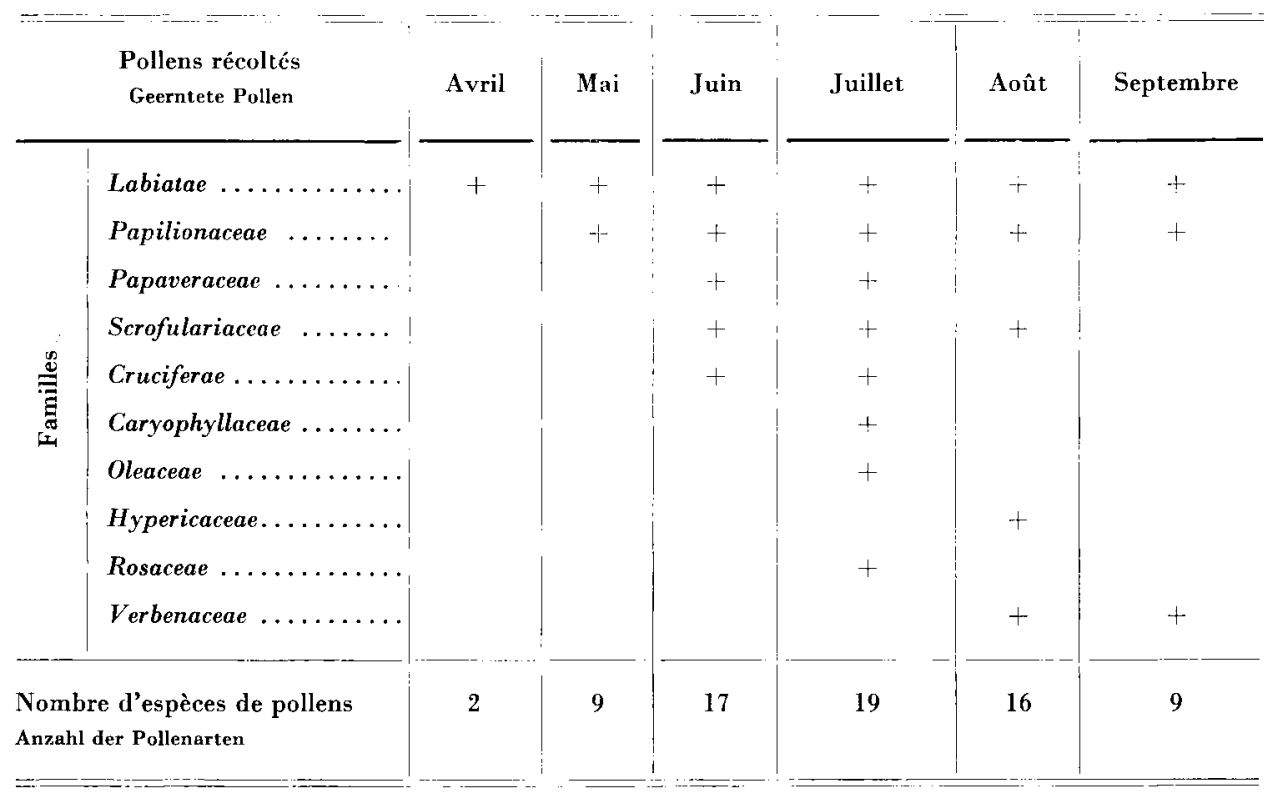




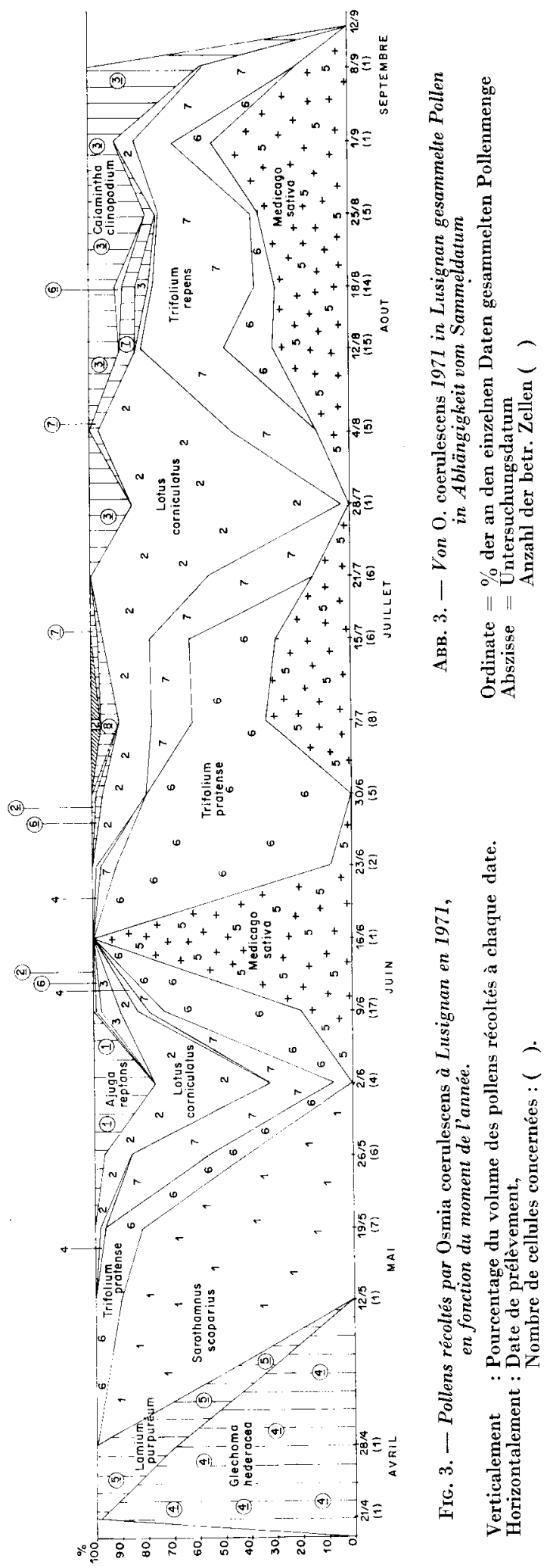




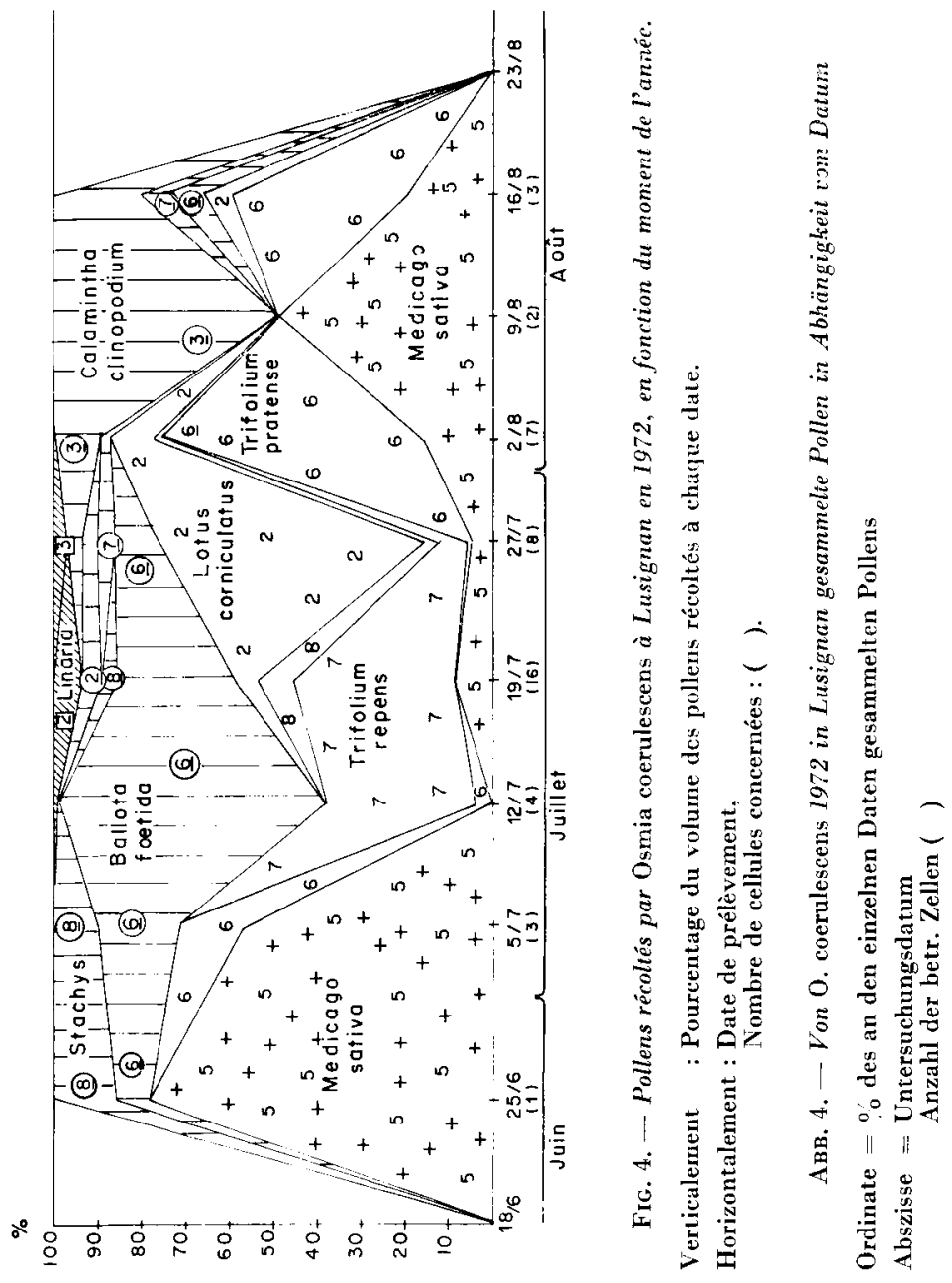



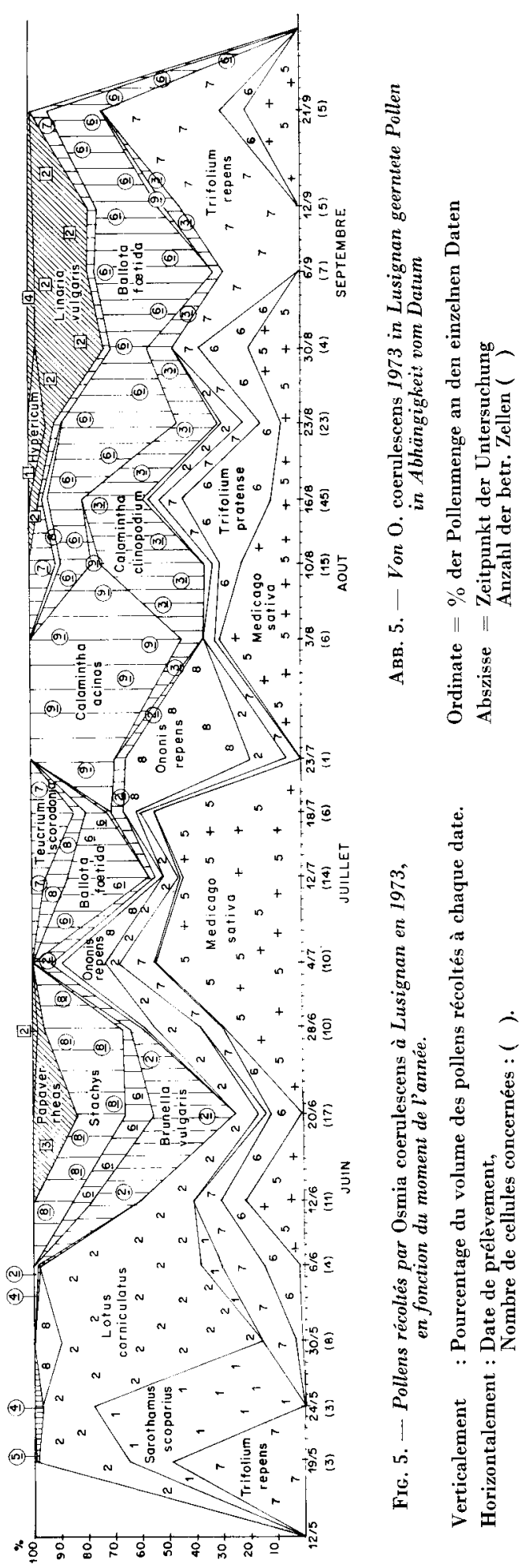
TABL. 6. - Nombre de cellules d'Osmia coerulescens renfermant des provisions uniflorales ou des associations de pollens.

ТАв. 6. - Anzahl der Zellen von 0. coerulescens, die einheitliche Pollenvorräte oder Mischungen verschiedener Pollenarten enthalten.

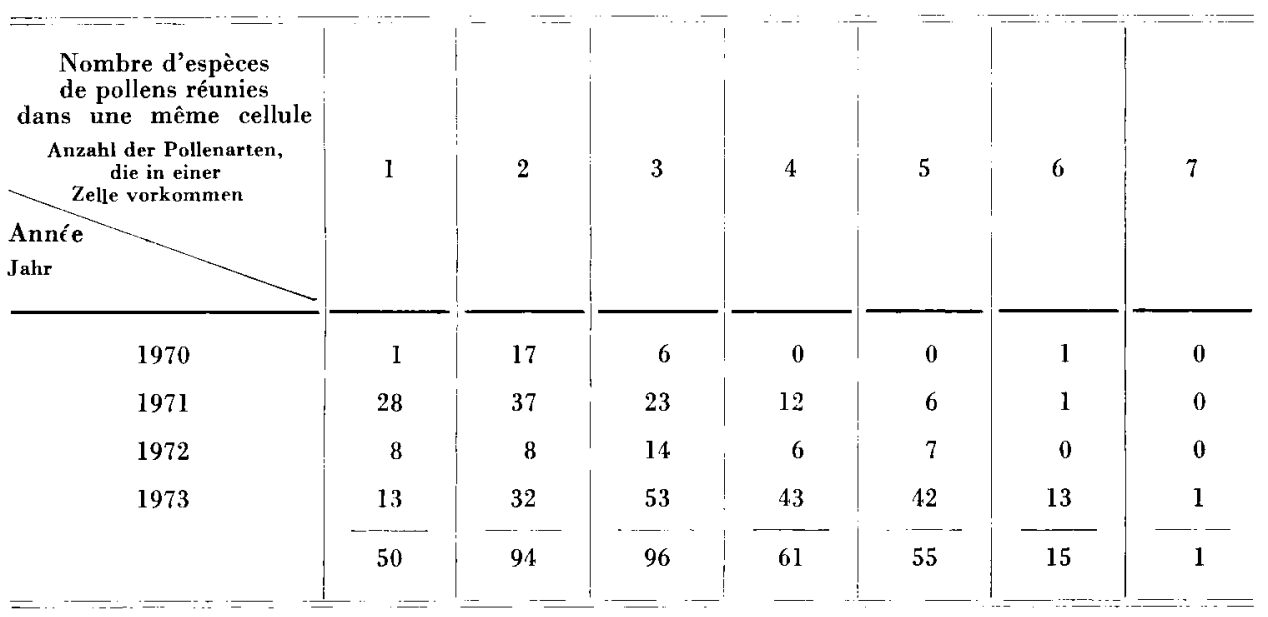

Les provisions uniflorales contiennent les pollens principaux : Trifolium repens, $T$. pratense, Lotus corniculatus, Medicago sativa, Sarothamnus scoparius, Ballota foetida (tabl. 7).

Il est à noter qu'au cours de la saison les Labiées ne sont pas toujours présentes, par contre les Papilionacées le sont dans 370 provisions sur 373 .

TABL. 7. - Nombre et contenu des cellules d'Osmia coerulescens à provision uniflorale sur un total de 373 cellules.

TAв. 7. - Anzahl und Inhalt von Osmia- Zellen mit einheitlichem Polleninhalt von insgesamt 373 Zellen.

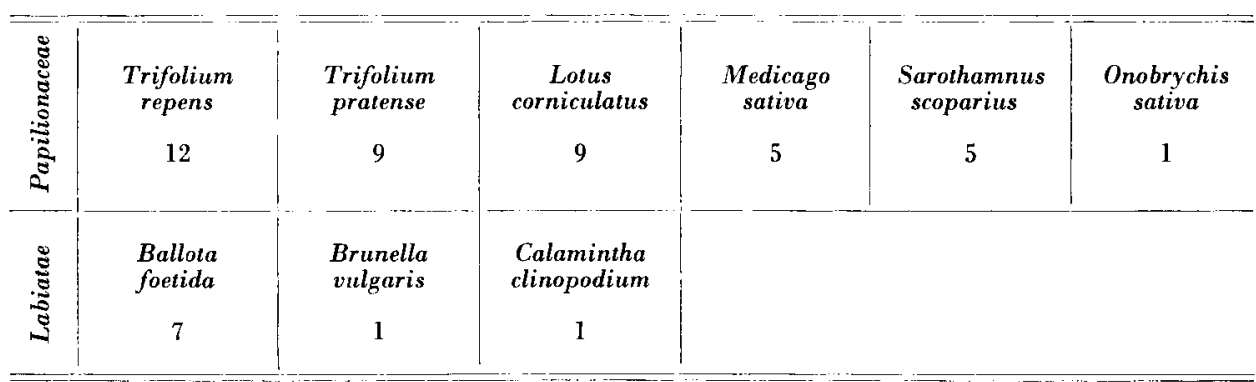



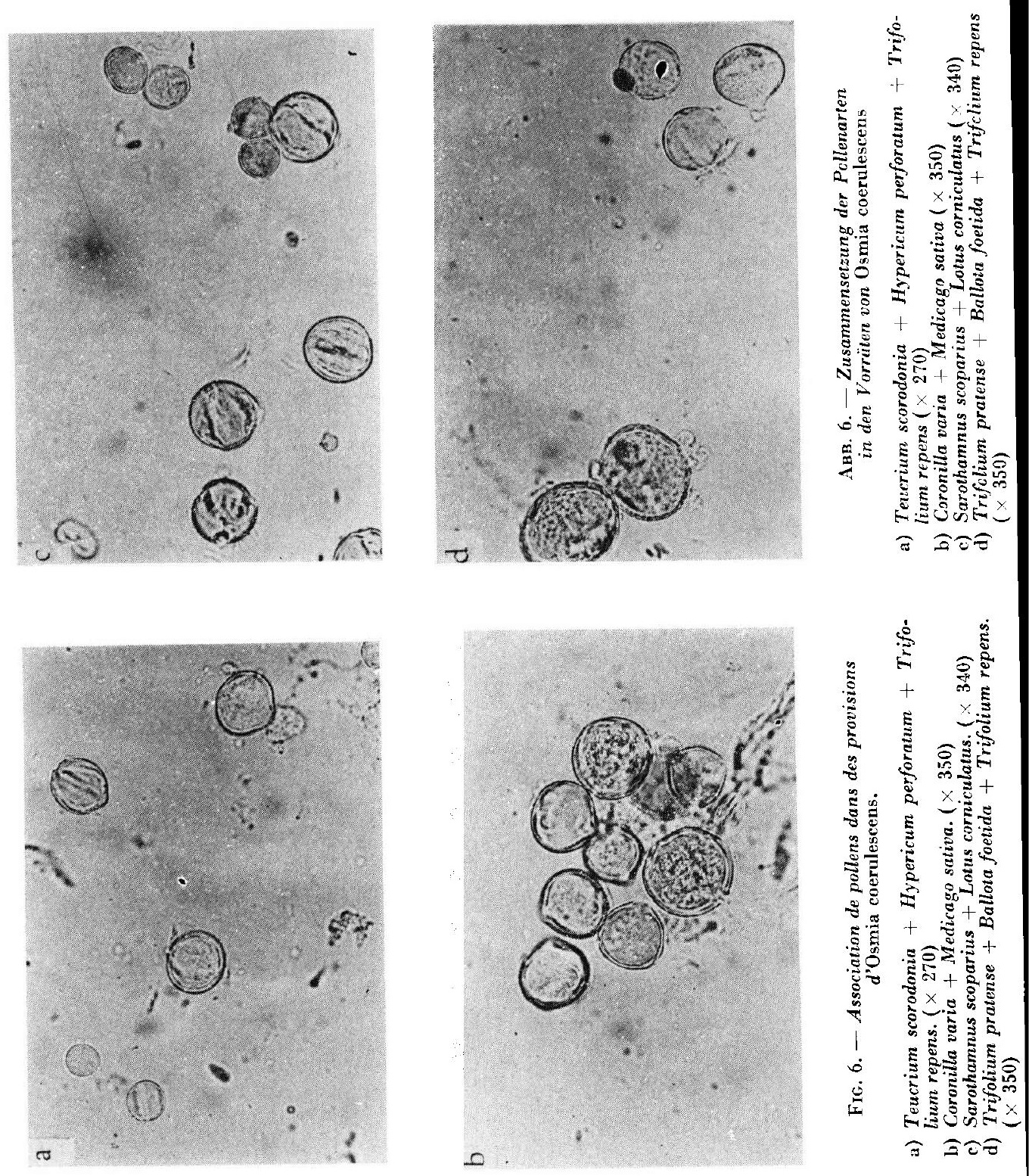

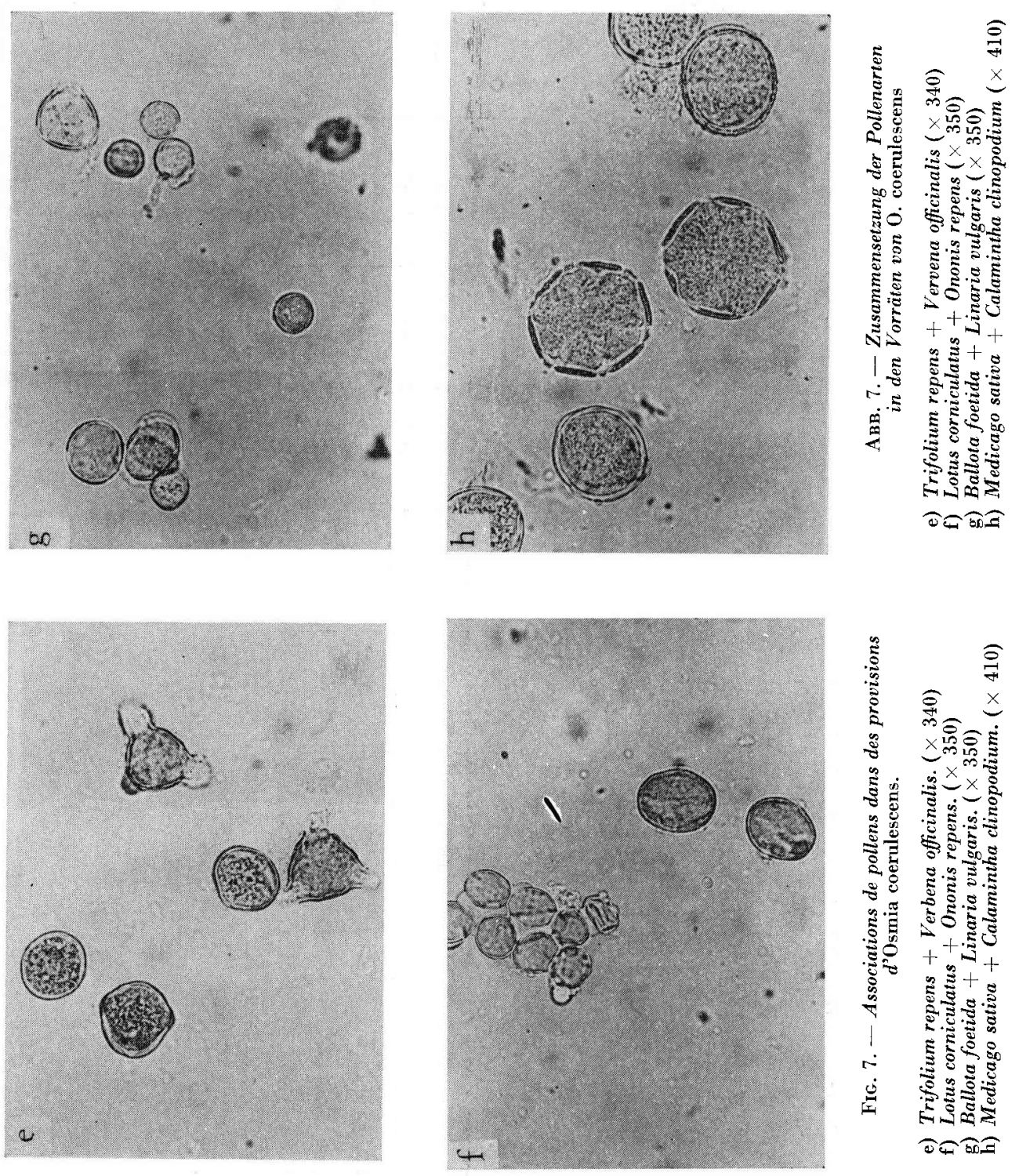


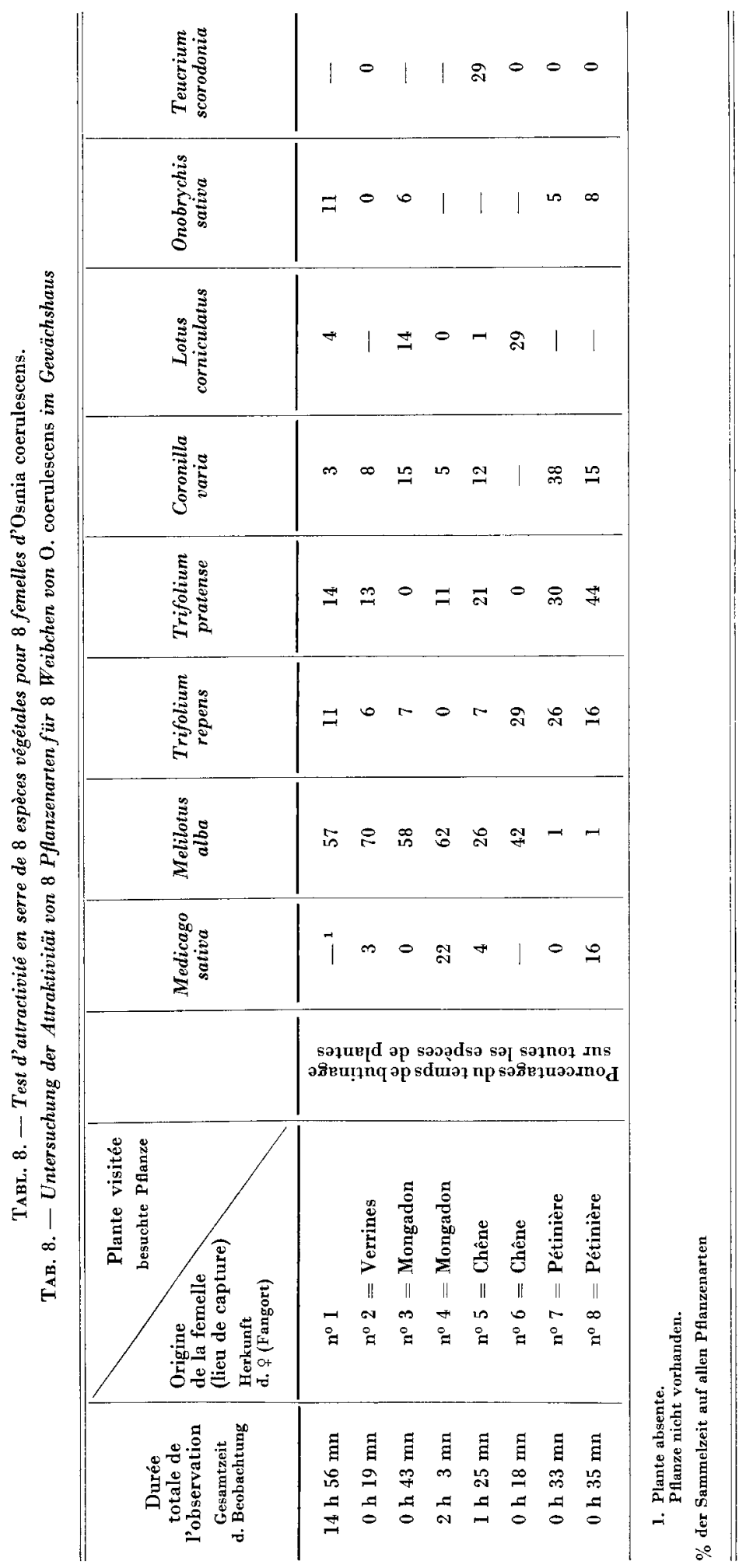




\section{II. - Attractivité comparée de quelques espèces florales}

Le tableau 8 présente les résultats de tests d'attractivité de 8 espèces florales (7 Papilionacées et 1 Labiée). Une interprétation précise n'est pas possible car si certaines femelles sont observées pendant un temps assez long (individus $n^{\circ} 1, n^{\circ} 4, n^{\circ} 5$ ) les autres ne font l'objet que d'un ou deux contrôles de butinage. Par ailleurs il $n$ 'a pas toujours été possible de fournir aux abeilles toutes les espèces de plantes. Enfin le nombre de femelles issues du même lieu de capture est faible. Malgré les imperfections de cette étude quelques remarques peuvent être faites :

- Dans des conditions de concurrence idéale, le Mélilot semble attirer les osmies d'une façon très sensible et la Luzerne a tendance à être délaissée au profit du Mélilot, des Trèfles et de la Coronille.

- Le Teucrium n'est visité que par une osmie sur cinq.

- Deux femelles de même origine (“ Pétinière 》) se comportent très différemment par rapport aux autres : elles ne visitent pratiquement pas le Mélilot et affectionnent surtout les Trèfles et la Coronille.

La Figure 8 rend compte de la relation existant entre le temps de butinage et le volume de pollen récolté chez 4 espèces. Il y a une forte corrélation entre les 2 données ( $\propto \mathbf{r}$ ) significatif à 1 pour cent dans 3 cas). Chez la Luzerne, le Trèfle blanc et le Melilot la pente de la droite de régression est égale à 1 environ $(0,8$ n'est pas significativement différent de 1$)$. Ce qui veut dire qu'il y a une bonne concordance entre les proportions des temps de butinage et celles des quantités de pollen récolté. Dans le cas du Trèfle violet les femelles auraient tendance à récolter, pendant des temps de butinage égaux, plus de pollen que chez les 3 autres espèces (La pente de la droite n'est significativement différente de 1 qu'avec un risque d'erreur de 20 pour cent).

\section{III. - L'activité d'approvisionnement}

Lorsqu'une femelle approvisionne une cellule son temps se décompose en butinage, travail dans la cellule, et parfois repos. Sur le tableau $n^{\circ} 9$ on constate que 3 femelles observées pendant plus de 20 heures passent en moyenne plus des $3 / 4$ de leur temps à butiner ( 74 à 87 pour cent du temps total) pour 8 à 21 pour cent à travailler à l'intérieur de la cellule. Quant au temps de repos il peut atteindre 14 pour cent.

On a effectué 9 contrôles de l'activité d'une de ces femelles (tabl. no 10). Ces contrôles échelonnés sur 10 jours montrent la relative constance du comportement d'un individu : 78 à 93 pour cent du temps sont consacrés au butinage et 6 à 12 pour cent au travail dans la cellule. On note une légère augmentation des temps de repos en fin de période (jusqu’à 15 pour cent). 

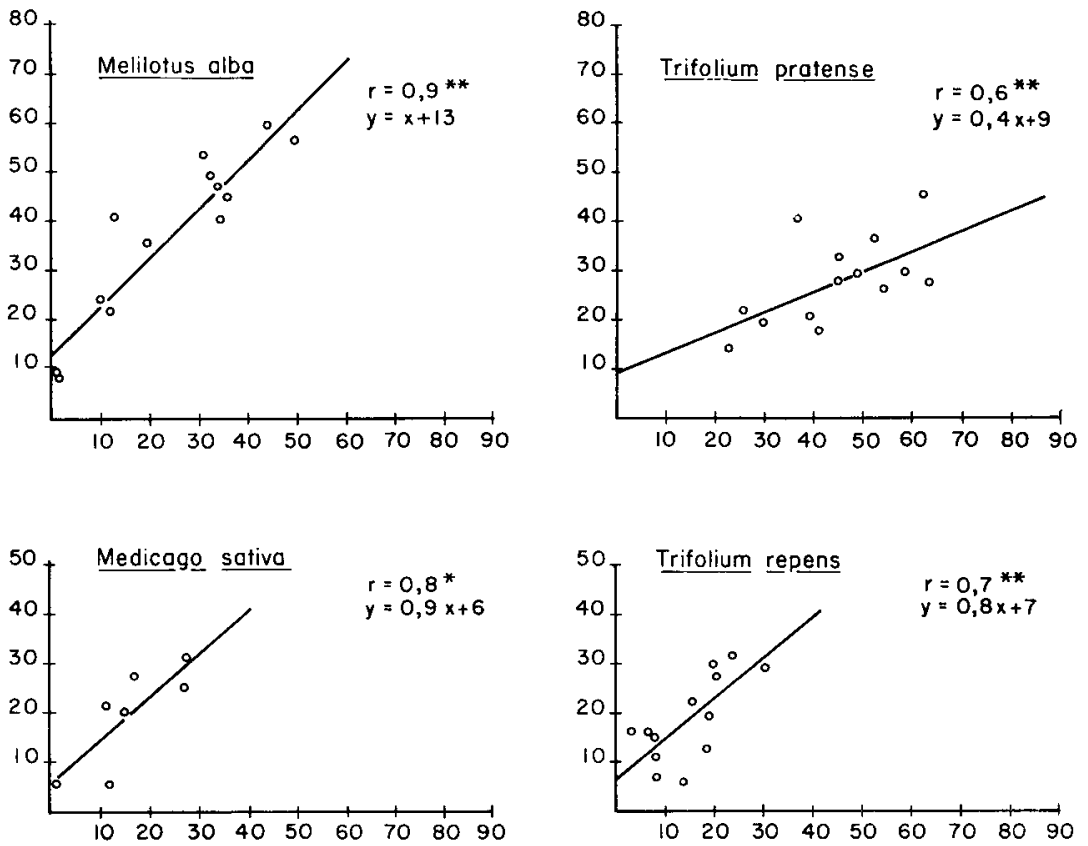

FIG. 8. - Relation entre le temps de butinage d'Osmia coerulescens et le volume de pollen récolté en fonction de l'espèce visitée.

Verticalement : Temps de butinage en pourcentage du temps total.

Horizontalement : Volume de pollen récolté en pourcentage du volume total des pollens récoltés. $\times$ signification à $5 \%$ d'erreur

$\times \times$ signification à $1 \%$ d'erreur.

Aвв. 8. - Beziehung zwischen der Sammelzeit von 0 . coerulescens und der eingetragenen Pollenmenge in Abhängigkeit von der besuchten Pflanze

Ordinate $=$ Eingetragene Pollenmenge in \% der Gesamtpollenmenge

Abszisse $=$ Geerntete Pollenmenge in $\%$ der Gesamtmenge der geernteten Pollen $\times$ bedeutet bis $5 \%$ Fehler

$x \times$ bedentet bis $1 \%$ Fehler.

TABL. 9. - Activité générale de 3 femelles d'Osmia coerulescens en serre pendant l'approvisionnement de leur nid.

TAB. 9. - Haupttätigkeit von drei Osmia- Weibchen im Gewächshaus während der Verproviantierung ihrer Nester.

\begin{tabular}{|c|c|c|c|c|}
\hline & \multicolumn{3}{|c|}{$\begin{array}{c}\text { Pourcentage du temps total } \\
\text { d'observation } \\
\text { Prozentsatz der Gesamt- } \\
\text { Beobachtungszeit }\end{array}$} & \multirow{2}{*}{$\begin{array}{l}\text { Temps total } \\
\text { d'observation } \\
\text { Gesamtzeit } \\
\text { der Beobachtung }\end{array}$} \\
\hline & $\begin{array}{l}\text { Butinage } \\
\text { Sammel- } \\
\text { tätigkeit }\end{array}$ & $\begin{array}{l}\text { Séjour } \\
\text { dans le nid } \\
\text { Aufenthalt } \\
\text { im Nest }\end{array}$ & $\begin{array}{c}\text { Repos } \\
\text { Ruhe }\end{array}$ & \\
\hline Femelle A (1970) . . . . . & 74 & 12 & 14 & $2 \mathrm{~h} 20 \mathrm{mn}$ \\
\hline Femelle B (1971) ....... & 79 & 21 & 0 & $0 \mathrm{~h} 54 \mathrm{mn}$ \\
\hline Femelle C (1971) . . . . . . & 87 & 8 & 5 & $17 \mathrm{~h} 48 \mathrm{mn}$ \\
\hline
\end{tabular}


Tabl. 10. - Vieillissement de la Femelle « $C » d$ 'Osmia coerulescens el constance de san activité en serre.
(a) = matin
(b) = après-midi.

TAB. 10. - Altern des Weibchens « $C$ » (Osmia coerulescens) und seine Ausdauer bei der Tätigkeit im Gewächshaus.

(a) $=$ Morgen

(b) = Nachmittag.

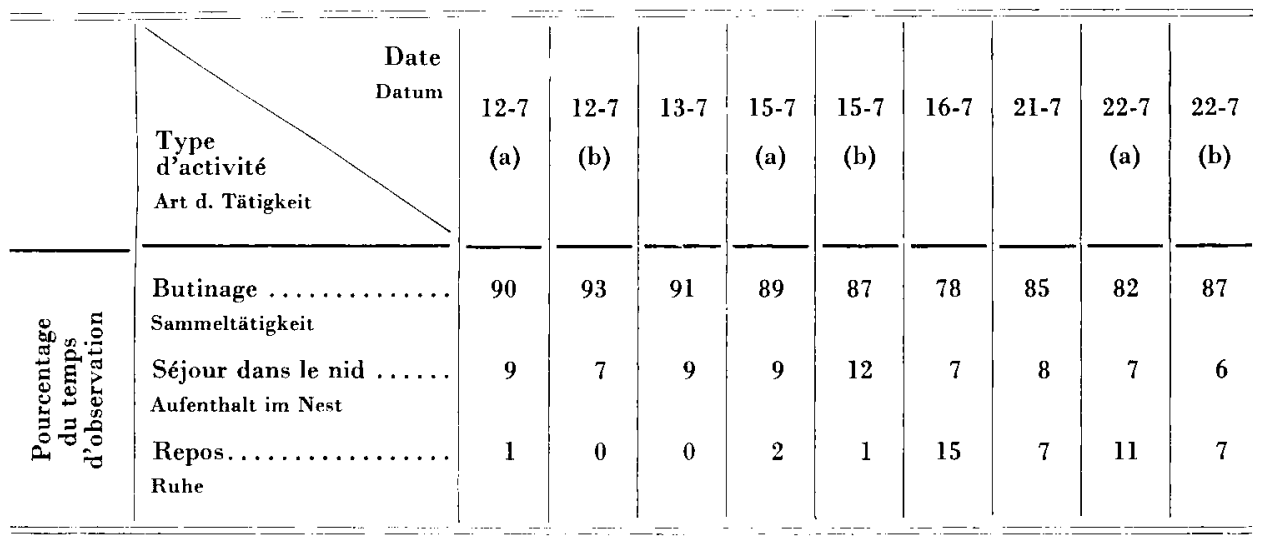

Cette même femelle ( $(\mathrm{C})$ ) fait des voyages de butinage de plus de 9 mn en moyenne. La variabilité des durées de voyage est grande (fig. 9 A) puisque la plus brève est de $1 \mathrm{mn} 30 \mathrm{~s}$ et la plus longue de $24 \mathrm{mn}$. Les séjours au nid sont décomposables en 2 actions : dans un premier temps la femelle malaxe ses provisions, régurgite du nectar et probablement y incorpore des sécrétions glandulaires. Puis elle sort du nid et rentre à nouveau à reculons la tête tournée vers la sortie, ceci lui permet de déposer le pollen en grattant sa brosse avec ses pattes postérieures. La première séquence dure en moyenne 23 s (10 à $50 \mathrm{~s}$ ) la seconde $34 \mathrm{~s}$ ( 10 à $80 \mathrm{~s}$ ) (fig. $9 \mathrm{~B}$ et $\mathrm{C}$ ).

\section{DISCUSSION}

\section{Sur les techniques}

Parmi 22 publications que j'ai pu consulter, concernant l'étude du butinage chez les osmies, seulement 11 mentionnent la technique employée. Le plus souvent l'inventaire des fleurs visitées est établi à partir des captures d'abeilles mâles ou femelles, mais dans certains cas l'auteur procède à l'analyse des pollens ramenés au nid : Rust et CLÉment (1972) prélèvent le pollen dans la brosse ventrale d'Osmia glauca Fowler et d'O. nemoris Sand. tandis que FreE 

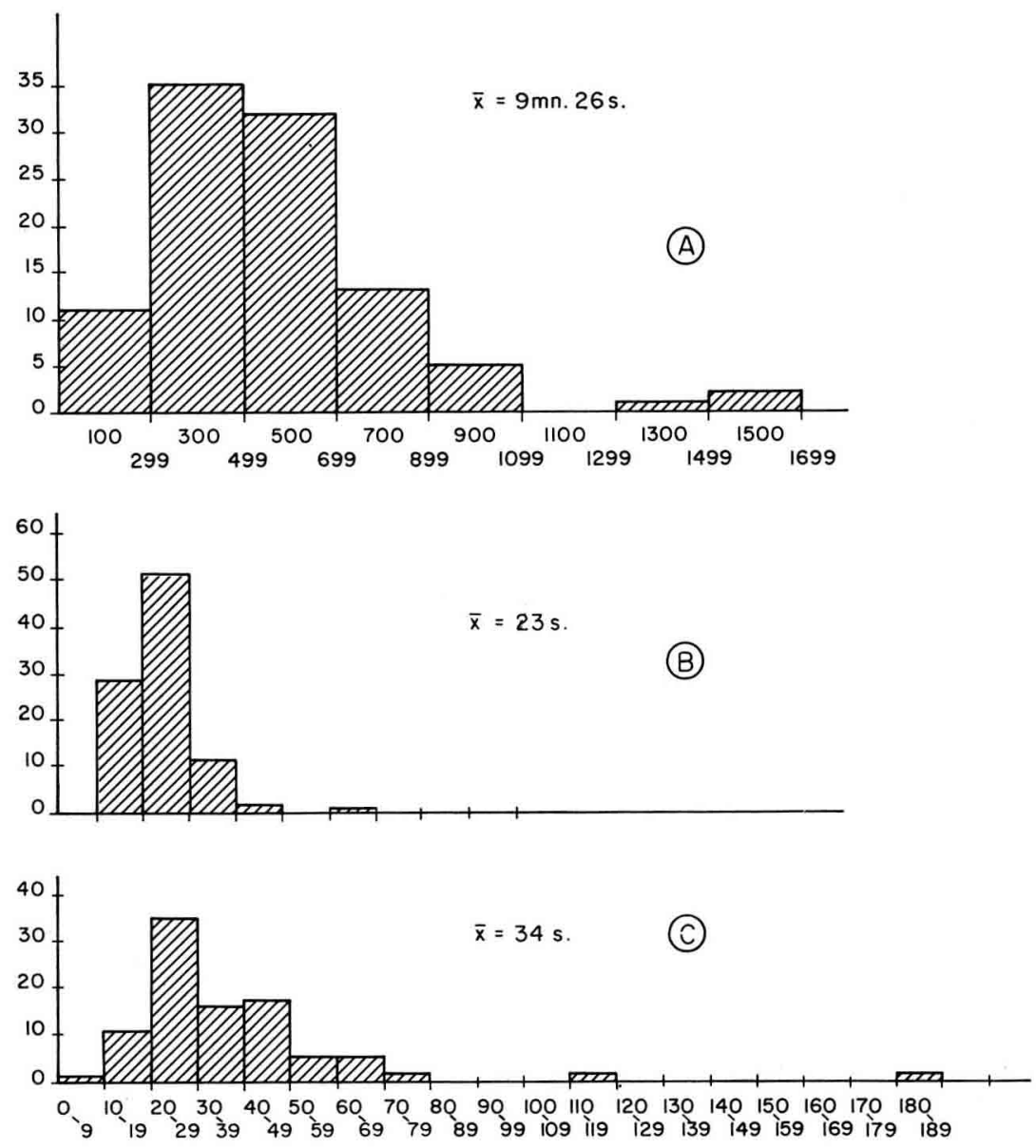

FIG. 9. - Variabilité de la durée de 3 séquences d'approvisionnement des cellules chez Osinia coerulescens (femelle $C$ ).

A - Visite des fleurs.

B - Régurgitation du nectar (position : tête dans la cellule).

$\mathrm{C}$ - Déchargement du pollen (position : abdomen dans la cellule).

Verticalement : Fréquence.

Horizontalement : Durées en secondes.

AвB. 9. - Veränderlichkeit der Dauer dreier Folgen der Verproviantierung der Zellen bei O. coerulescens (Weibchen $C$ )

$\mathrm{A}=$ Blïtenbesuch

$\mathrm{B}=$ Auswärgen des Nektars (Stellung: Kopf in der Zelle)

$\mathrm{C}=$ Abladen des Pollens (Stellung : Abdomen in der Zelle)

Ordinate $=$ Häufigkeit

Abszisse = Dauer in Sekunden

et Williams (1970) et Rust, Thorp et Torchio (1974) analysent le pollen des excréments larvaires chez $O$. rufa $\mathrm{L}$. et $O$. nigrifrons Cress. respectivement. Une troisième technique parfois complémentaire de la précédente, consiste à analyser les provisions non consommées dans les cellules; c'est ainsi qu'ont 
travaillé Rust et Cíf́ment (1972), Free et Williams (1970), Brian (1951) et Krombein (1969) chez O. latreilli Spin. et O. aurantiaca Stan.

Dans le cas d' $O$. coerulescens la seule technique utilisée est l'analyse des provisions non dévorées. On ne peut donc connaître le comportement de butinage des mâles et les résultats ne permettent pas d'affirmer que les plantes fournissant le pollen aux femelles sont les seules plantes visitées. Par ailleurs, l'analyse hebdomadaire des nids et la surveillance des flores locales environnant les sites choisis facilitent beaucoup les déterminations et augmentent leur précision.

On remarque la relation existant entre la richesse d'un spectre et la taille de l'échantillon de provisions. Une trentaine de cellules échantillon doit donner une précision suffisante pourvu que leur récolte soit échelonnée tout au long de la période d'activité de l'espèce étudiée et ceci pendant 3 ans.

\section{Sur les résultats}

On trouve peu de renseignements sur le butinage d' $O$. coerulescens dans la littérature : FrIEse (1911) indique que cette espèce visite Ajuga et Lotus Creveceur (1925) Ajuga, Lecherco (1942) Marrubium vulgare, Chandeer (1963) Leonurus et Nepeta, Pesenko (1971) les Labiées et les Papilionacées. Seul Raw (1974) affirme que cette osmie récolte du pollen de Composées en grande quantité ce qui laisse supposer qu'il y a eu confusion entre 2 espèces.

Le spectre pollinique d' $O$. coerulescens établi dans une région limitée n'a, dans le détail, qu'une valeur limitée. Cependant il est très probable que des études du même type réalisées dans d'autres régions et d'autres pays montreraient que cette espèce d'osmie a une grande attirance pour la famille des Labiées et surtout celle des Papilionacées. Son comportement de butinage est moins polylectique que celui d'O. cornuta et $O$. rufa (TASEI, 1973) récoltant le pollen de 8-9 familles botaniques, mais il est moins oligolectique que celui d'O. californica butinant la seule famille des Composées (Levin, 1966).

Le choix des pollens se fait selon certaines tendances de butinage au sein de l'éventail de plantes fleurissant au moment du vol des femelles dans un rayon d'environ $100 \mathrm{~m}$ autour du site de nidification. Deux tendances additionnent leurs effets : la tendance héréditaire à butiner telle ou telle plante et l'attirance pour les espèces florales les plus abondantes.

L'étude que j'ai présentée ici ne peut pas donner l'idée sur la variabilité des tendances héréditaires du butinage en fonction de l'origine géographique des abeilles.

Le comportement d'approvisionnement est caractérisé par des voyages de butinage relativement brefs : en moyenne $9 \mathrm{mn}$, alors qu'O. lignaria, $O$. californica Cress. et $O$. nigrifons passent 15 à $19 \mathrm{mn}$ sur les fleurs avant de 
retourner au nid (Leviv, 1966 - Rust, Thorp et Tonchio, 1974). D'autres espèces effectuent des voyages de butinage dans des temps moyens proches de ceux d'O. coerulescens : O. submicans Mor., O. rufa, O. excavata Afk. (Wafa, Eu Berry, 1971 - Tasei, 1973 - Hirashima, 1957). Les temps de séjour dans le nid, $57 \mathrm{~s}$, en moyenne sont aussi parmi les plus brefs si on les compare avec ceux d'O. californica (120 s).

\section{CONCLUSION}

Osmia coerulescens récolte une trentaine d'espèces de pollen. Bien que polylectique cette abeille solitaire a tendance à être inféodée aux Papilionacées (Luzerne, Trèfle blanc, Trèfle violet, Lotier) et secondairement aux Labiées (Calamintha, Ballota). Selon l'année considérée, 0 à 5 pour cent de la récolte de pollen sont composés de plantes appartenant à 8 autres familles principalement : Scrofulariacées, Papaveracées, Verbenacées, Hypéricacées. L'attirance de cette osmie pour le pollen de Medicago sativa est certaine (près de 19 pour cent du volume total). Cependant Melilotus alba semble bien être la plante la plus attractive de toutes.

Le spectre pollinique du mois de juillet est le plus riche (19 espèces). Les mois de mai et septembre ne permettent pas de récoltes aussi variées (9 espèces).

Dans chacun des sites de nidification le spectre pollinique présente souvent une association de 2 pollens caractéristiques, relativement abondants d'une année à l'autre.

Dans leur majorité les provisions polliniques renferment des mélanges de 2,3 et 4 espèces florales. La plupart des provisions uniflorales contiennent un pollen de Papilionacée.

L'approvisionnement se fait d'une manière rapide : un voyage de butinage dure en moyenne $9 \mathrm{mn}$ et un séjour à l'intérieur du nid $1 \mathrm{mn}$.

Les résultats montrent bien que le spectre pollinique d'une espèce d'Apoïde dans une région déterminée peut être établi après 3 ans d'échantillonnage. Une trentaine de provisions donnent une précision suffisante pourvu que les prélèvements soient effectués dans plusieurs biotopes et qu'ils soient répartis tout au long de la période d'activité de l'espèce. Si l'on possède un grand nombre d'échantillons, le spectre peut être obtenu de façon rapide en calculant simplement la fréquence des provisions contenant chaque espèce de pollen.

Une question reste posée : existe-t-il des variations dans le choix des pollens, dues à l'origine géographique des osmies; dans l'affirmative, est-ce dû à un conditionnement larvaire? Il sera possible d'y répondre en utilisant les techniques d'élevage en serre et de contrôle du butinage.

Reçu pour publication en mai 1976.

Eingegangen im Mai 1976. 


\section{REMERCIEMENTS}

Je tiens à exprimer ma reconnaissance envers M. BARon (Faculté des Sciences de Poitiers) qui a bien voulu identifier les plantes. Je remercie également le Dr VorwoHL (Institut d'Apiculture de Hohenheim) pour l'identification de pollens de Scrofulariacées et Hypericacées.

\section{ZUSAMMENFASSUNG}

Nach einer vorangegangenen Arbeit über die Biologie von Osmia coerulescens (TaseI, 1972) legt Verfasser nun die Ergebnisse seiner Beobachtungen über das Sammelverhalten dieser Art in der Gegend von Lusignan (Mittelwesten Frankreichs) vor.

\section{Material und Methoden}

1. 50 bis 120 zerlegbare Bambus-Nistanlagen wurden an verschiedenen Hauswänden und an Pfosten angebracht. Insgesamt wurden in vier Jahren (1970-1973) 373 wöchentliche Untersuchungen und Pollenanalysen durchgeführt (cf. : Mavrizio und Lovveaux, 1965) und zwar in den Monaten April bis September, der Zeit der Aktivität von Osmia.

2. Acht Weibchen wurden in Gewächshäusern gezogen, und während insgesamt 21 Stunden wurde ihr Sammelverhalten auf verschiedenen, gleichzeitig angebotenen blühenden Pflanzenarten beobachtet.

\section{Ergebnisse}

1. Osmia coerulescens sammelt Pollen auf $30 \mathrm{zu} 10$ Familien gehörenden Pflanzenarten (Tab. 1; Abb. 1). Zwei Familien herrschen vor : Labiaten und Papilionaceen. Sechs der 30 erwähnten Arten finden sich in mehr als $30 \%$ der Vorräte : Trifolium repens, T. pratense, Medicago sativa, Lotus corniculatus, Calamintha clinopodium, Ballota foetida. Pollen, die zu anderen Familien gehören, kommen nicht so häufig vor. Vier Arten von ihnen sind verhältnismässig gut vertreten : Linaria vulgaris (7\%), Papaver rhoeas $(4 \%)$, Verbena officinalis $(3 \%)$, Hypericum perforatum ( $3 \%$ ).

2. Unabhängig vom Beobachtungsjahr beträgt der Anteil der Papilionaceen stet; mehr als $50 \%$ des Gesamtvolumens des eingetragenen Pollens. Der Anteil der Labiaten beträgt durchschnittlich $31 \%$ der Pollenvorräte und der anderer Pflanzen $3 \%$ (Tab. 2).

3. Trotz der Veränderlichkeit der Pollenspektren sieht man auf Tab. 2, dass die Osmien die Neigung besitzen, an jedem Aufstellplatz in jedem Jahr die gleichen Pollen zu sammeln, z.B. Medicago sativa und Trifolium repens in " Verrines ", Trifolium pratense und Calamintha clinopodium in “ Pétinière ", Lotus corniculatus und Sarothamnus scoparius in “ Mongadon ".

4. Das Spektrum der Pollenvorräte ist in der Zeit von Juni bis August am reichhaltigsten, besonders im. Juli, wo 19 von 30 Pollenarten gesammelt werden können (Abb. 3, 4, 5 und Tab. 5). Papilionaceen und Labiaten sind von Mai bis September immer vertreten, während Hypericaceen, Verbenaceen und Rosaceen ihren Pollen nur am Ende der Saison den Weibchen der 2. Generation bieten.

5. Von 373 Pollenvorräten bestehen 306 aus einer Mischung von 2-5 Pollenarten (Abb. 6 u. 7); 16 enthalten 6-7 verschiedene Pollenarten und 50 weisen nur eine Pollenart auf (Tab. 6).

6. Im Treibhaus ist Melilotus alba die Pflanze mit der grössten Anziehungskraft. (Diese Art wächst in der Gegend von Lusignan nicht wild). Es besteht eine starke Korrelation zwischen der Sammelzeit auf einer Pflanzenart und der Menge des gesammelten Pollens (Abb. 8). 
7. Während der Versorgung des Nestes mit Pollen verbringen die Weibchen mehr als $3 / 4$ der Zeit auf den Blüten; die übrige Zeit wird in der Zelle verbracht oder dient der Ruhe (Tab. 10). Durchschnittlich dauert das Beladen der Bürsten mit Pollen 9 Minuten und der Aufenthalt im Nestinnern 1 Minute (Abb. 9).

\section{RÉFERENCES BIBLIOGRAPHIQUES}

Brian A. D., 1951. - The pollen collected by bumblebees. J. anim. Ecol. 20, 191-194.

Chander L., 1963. - Interspecific competition between two species of Osmia. Proc. North. centr. Branch E.S.A. $18,30$.

Crevecaur A., 1925. - Les osmies de la Belgique (G. Osmia Pz.). Ann. Soc. Entomol. Belg. Brussels, 65, 183-194.

Free J. B., Williams I. H., 1970. - Preliminary investigations on the occupation of artificial nests by Osmia rufa L. (Hym. Megachilidae) J. Appl. Ecol. 7, 559-566.

Friese H., 1911. - Apidae. I. Das Tierreich 28 Lieferung-Megachilinae. Berlin.

Hirashima Y., 1957. - Further observations on the life-history and habits of Osmia excavata Alfk. (Hym. Megachilidae). Fac. Agric. Kyushu Univ. Sci. Bull. 16, 193-202.

Holm S. N., 1973. - Osmia rufa L. (Hym. Megachilidae) as a pollinator of plants in green houses. Entomol. Scond., 4, 217-224.

Krombein K. V., 1969. - Life history notes on some Egyptian solitary bees and wasps and their associates. Wash. Smithsonian Inst. Press, 19, $18 \mathrm{p}$.

LeclercQ J., 1942. - Notes sur les Hyménoptères. Observations sur le choix des fleurs butinées. Ann. Soc. Royale Zool. Belgique, 73, 1-10.

Levin M. D., 1966. - Biological notes on Osmia lignaria and Osmia californica. J. Kans. Entomol. Soc. 39, 524-535.

Maurizio A., Louveaux J., 1965. - Pollens de plantes mellifères d'Europe. U.G.A.F. 38, Bd Sébastopol. Paris.

Pesenko Y. A., 1971. - On the fauna and ecology of Apoidea (Hymenoptera) of the steppes of the lower Don. I. The family Megachilidae. Entomol. Rev. 50, 38-47.

Raw A., 1974. - Pollen preferences of three Osmia species (Hymenoptera) Oikos, 25, 54-60.

Rust R. W., Clement S. L., 1972. - The biology of Osmia glauca and Osmia nemoris (Hym. Megachilidae). J. Kans entomol. Soc. 45, 523-528.

Rust R. W., Thorp R. W., Torcho P. F., 1974. - The ecology of Osmia nigrifrons with a comparison to other Acanthosmioides. J. nat. Hist., 8, 29-47.

Taser J. N., 1972. - Observations préliminaires sur la biologie d'Osmia (Chalcosmia) coerulescens L. (Hym. Megachilidae) pollinisatrice de la luzerne. Apidologie, 3, 149-165.

TAsei J. N., 1973, - Le comportement de nidification chez Osmia (Osmia) cornuta Latr. et Osmia (Osmia) rufa L. (Hym. Megachilidae). Apidologie, 4, 195-225.

Tовсніо P. F. - Communication personnelle.

WAFA A. K., El Berry A. A., 1971. - Some biological aspects of two local wild bees with reference to the immature stages of Osmia submicans Mor. (Hym. Megachilidae). Bull. Soc. Entomol. Egypte, 55, 379-392.

Yamada M., Oyaya N., Sekita N., Shirasaki S., Tsugawa C., 1971. - Preservation and utilization of natural enemies and useful insects in apple orchards. III. The ecology of the megachilid bee, Osmia cornifrons Rad. (Hym. Apidae) and its utilization for apple pollination. Bull. Aomori apple Exp. Sta., 15, 80 p. 